\title{
SÍNTESIS DIALÓGICA PARA LA FORMACIÓN EN ARQUITECTURA*
}

Jemay Mosquera Téllez***

Ernesto León Duplat Upegui****

Grupo de Investigación Gestión Integral del Territorio - GIT

Universidad de Pamplona, Colombia

Recibido: I I octubre 201 I

Aprobado: 29 noviembre 2011

Articulación de los procesos misionales

institucionales. Fuente: suministrada por los autores

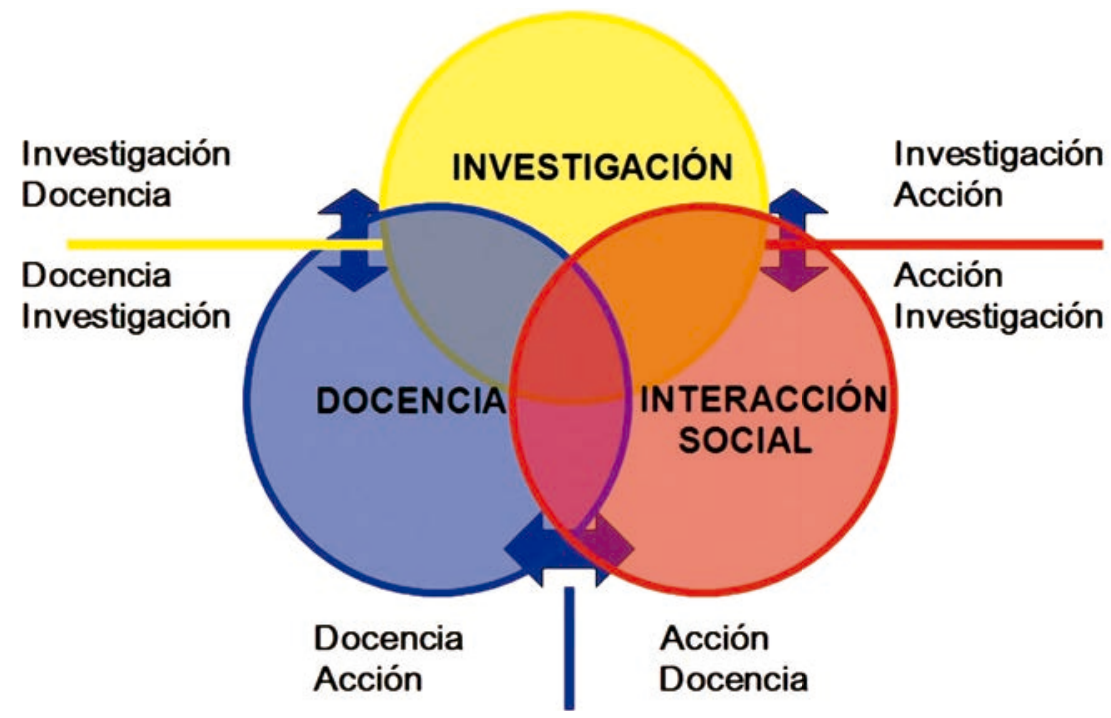

\section{RESUMEN}

El trabajo se soporta en una conceptualización previa en torno al desarrollo del conocimiento, la arquitectura y la formación disciplinar, la cual sirve de base para plantear una síntesis integradora adaptativa representada en una articulación dialógica, sistémica y didáctica de los elementos que interactúan en los procesos de diseño. Frente a la evidente diversidad histórica y espaciotemporal, la propuesta pretende confirmar el carácter transdisciplinar de las configuraciones arquitectónicas en contextos específicos de aplicación y aportar a la fundamentación de principios universales y uniformidades empíricas, así como a la formación integral de arquitectos que lideren procesos de desarrollo integral del territorio.

\section{PALABRAS CLAVE}

Adaptativa, dialógica, didáctica, sistémica y transdisciplinar. 


\title{
DIALOGIC SYNTHESIS FOR ARCHITECTURAL EDUCATION
}

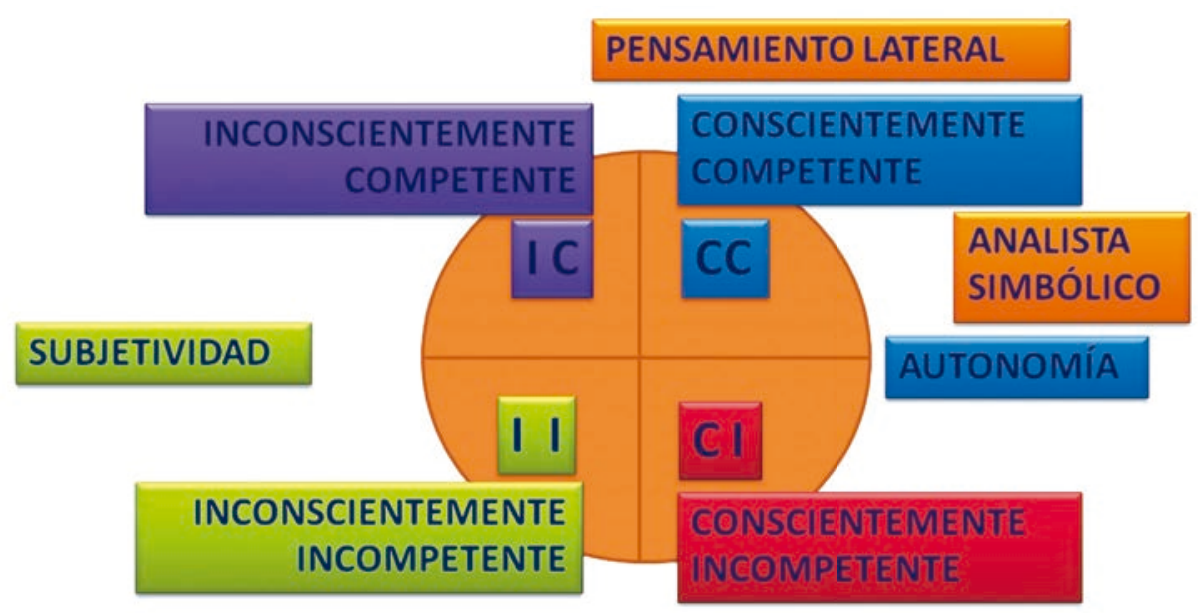

Niveles de competencia consciente. Fuente: suministrada por los autores.

\begin{abstract}
The paper is supported by a previous conceptualization around knowledge development, architecture and disciplinary training, which serves as a basis for raising an integrative and adaptive synthesis represented in a dialogical, systemic and didactic joint of the elements that interact in the design processes. Faced with the evident historical and spatiotemporal diversity, the paper aims to confirm the transdisciplinary nature of architectural configurations in specific contexts of application and contribute to the foundation of universal principles and empirical uniformities and also to the formation of architects to lead processes of integral land development.
\end{abstract}

\section{KEY WORDS}

Adaptive, dialogical, didactic, systemic, transdisciplinary.

Arquitecto de la Universidad de Pamplona (2007). Director y profesor de tiempo completo del programa de Arquitectura (20I I-a la fecha) de la Universidad de Pamplona en Villa del Rosario. Miembro del Grupo de Investigación Gestión Integral del Territorio - GIT de la Universidad de Pamplona y Editor de la revista Soluciones de Diseño del programa de Arquitectura de la Universidad de Pamplona.grupo_git@unipamplona.edu.co 


\section{INTRODUCCIÓN}

Los acelerados procesos de urbanización y las condiciones de crecimiento caótico de las ciudades, la destrucción acelerada y continua del entorno natural, el nuevo orden económico y las actuales tendencias de producción y comercialización, entre otras megatendencias, exigen nuevos métodos de estudio crítico y evaluación comparada del desarrollo y nuevos procesos de formación integral en arquitectura. Dichos métodos y procesos se enmarcan en una concepción integradora (física, biológica y antropológica) de la relación ser humano-naturaleza', en la velocidad de distribución de la información y en la articulación de esfuerzos en redes globales, para la generación de propuestas enfocadas al desarrollo de un conocimiento heterogéneo, socialmente útil y aplicado a contextos concretos.

El presente trabajo pretende hacer un aporte a las bases teóricas y prácticas de una formación integral en arquitectura y es fruto de resultados obtenidos en el grupo de investigación Gestión Integral del Territorio - GIT (dentro de la línea de investigación Pedagogía e investigación participativa) de la Universidad de Pamplona.

\section{INTERRELACIONES Y COMPLEMENTARIEDADES}

El espacio y el tiempo, como categorías investigativas fundamentales del hábitat, adquieren un especial significado en el estudio de la arquitectura, dado que cada hecho, norma, edificación, o proceso de configuración espacial ineludiblemente se inscribe en este sistema de coordenadas. ${ }^{2}$ Un aporte a las relaciones espacio temporales fue desarrollado por Mijail Bajtín (1895-1975), quien expuso una multiplicidad de significados relacionados con la diversidad cultural de cada comunidad y a partir del cual se pudo concebir la articulación tiempo y espacio de forma permanente, como un instrumento de análisis dialógico situado entre la poética y la retórica, la realidad histórica y el hecho arquitectónico, que mide y hace inteligible y de qué manera una obra representa la realidad. ${ }^{3}$ Por lo tanto, el estudio y la interpretación de los signos de dicha diversidad en el espacio y el tiempo se convierte en la base del diálogo, estimulan la necesidad de comprensión de las diferentes manifestaciones socio-culturales y la sensibilidad estético-emocional que permite el acercamiento entre diferentes culturas $y$ generaciones, entre individuos $y$ colectividades, $y$, por ende, la naturaleza científica, artística y ético-política del ser humano. ${ }^{4}$

A través de la historia de la arquitectura se puede observar cómo se ha empleado el apoyo de diversos saberes y campos de conocimiento específico para lograr, de manera eficiente, la articulación entre los conceptos de arte, entendido como búsqueda de la belleza y la ciencia, entendida como el desarrollo de procesos racionales, sustentados en la investigación y la experimentación ${ }^{5}$. Dichos conceptos se fundamentan en la reflexión en torno al desarrollo y la interacción de la práctica y la teoría.

La teoría abarca el arte, las reglas heredadas con las tradiciones y la ciencia demostrada por fórmulas, mientras que la práctica es la perfecta adecuación de la teoría a los materiales, el clima y las condicionantes de cada contexto ${ }^{6}$. De esta manera, se puede observar que la

MORÍN, Édgar, CIURANA, Emilio y MOTTA Raúl. Educar en la era planetaria. El pensamiento complejo como un método de aprendizaje en el error y la incertidumbre humana. I ${ }^{a}$. Ed. España, U de Valladolid, Editorial UNESCO, 2003. Pp. 38-80.

2 MOSQUERA, Jemay. Síntesis dialógica para la formación en arquitectura. Memorias 5 Seminario Internacional Arquitectonics Network en América, the Project as research, research as Project, ISSN 978-958-8477-32-9. Universidad Santo Tomás, Bucaramanga. 2012, p. 24

3 BAJTIN, Miajil. Las formas de tiempo y del cronotopo en la novela, ensayos de poética histórica. Teoría y estética de la novela; Madrid, Taurus, 1989, pp. 237-409.

4 MUNTAÑOLA, Josep. Arquitectura, educación y dialogía social. En Revista española de de pedagogía, año LXII. No. 228, Universidad Politécnica de Catalunya, España. 2004, pp 223-227

5 SALDARRIAGA, Alberto. "Aprender Arquitectura. Un manual de supervivencia" En: Colombia 1966. Ed. Fundación Corona ISBN: 958-9054-4 v. 1996. p 22

6 Reconceptualización de la concepción arquitectónica del arquitecto restaurador parisino del siglo XIX Eugene Viollet-Due. 
práctica se relaciona con el continuo hacer de la arquitectura y el desarrollo de su campo profesional y la teoría como la manera reflexiva y argumentativa de explicar y sustentar los diversos resultados obtenidos en dichos desempeños.

Estas fuentes de conocimiento -práctica y teoría- se rigen históricamente de saberes de la arquitectura que son reconocidos por componentes, tales como utilidad, solidez y belleza ${ }^{7}$, los cuales han permeado la enseñanza teórica de la arquitectura y los desarrollos conceptuales de los hechos arquitectónicos.

La utilidad es atribuida a la función utilitaria del objeto arquitectónico como su principal razón de creación al servicio del ser humano y de las necesidades reales de la comunidad; la solidez se refiriere principalmente a ideas relacionadas con la firmeza y la estabilidad de la construcción, materialización o configuración de hechos tangibles o intangibles y la Belleza nos evoca al análisis del gusto, de lo bello y lo espontáneo en un amplio conjunto de sensaciones internas del ser humano. Las características de estos y los términos en relación con los saberes propios de la arquitectura, conforman una serie de conceptos que a partir de sus epistemologías se enmarcan dentro de los conceptos filosóficos relacionados con el ser humano, la tecnología y la belleza/gusto/deleite los cuales, a su vez, confluyen en el proyecto arquitectónico como síntesis de humano, lo tecnológico, lo ético, lo lógico y lo estético, en la que el arquitecto se apropia de su medio natural para transformarlo y emplearlo de manera adecuada y consciente para el uso de diferentes actividades humanas.

Estos conceptos contribuyen a la reflexión del proyecto de lo arquitectónico como objeto de análisis e interpretación desde diferentes posibilidades objetivas y subjetivas de entendimiento y asumir la arquitectura como disciplina que desde una reflexión ética, humanista y social, ayuda a optimizar la realización de las diversas actividades humanas mediante la configuración de ambientes que hacen más atractivas y útiles las actividades de convivencia, de trabajo y recreación y, al mismo tiempo, proporcionan placer estético y emocional. ${ }^{8}$

En ese proceso histórico del desarrollo de la arquitectura se han establecido diversas etapas (como práctica sin teoría, como expresiones de la práctica artística, como respuestas a las necesidades del hábitat, entre otras), estrechamente relacionadas con los procesos del desarrollo (la revolución agrícola, el renacimiento, revolución industrial y la sociedad del conocimiento) y los consecuentes cambios en otras disciplinas producto de dichos procesos. Tales posiciones, han influido en la alimentación de posturas antagónicas: la comprensión y configuración del espacio físico apoyada por conocimientos históricos, teóricos y artísticos generales y el saber técnico que ayuda a satisfacer las necesidades humanas y se apoya en una formación técnico-científica vinculada y aplicada en el contexto local.

Los nuevos retos que plantean las actuales condiciones de trasmisión, acumulación y generación de conocimiento están relacionados con la necesidad de asumir la interrelación sistémica de los hechos que se dan en el entorno y de buscar la integración de conocimientos y habilidades transversales para la arquitectura y la transformación consciente del territorio.

7 Componentes propuestos por el arquitecto Romano Vitruvio durante el siglo I d. C., mediante los conceptos de "Utilitas, Firmitas y Venustas"; los cuales a finales del siglo XV fueron debatidos por el Arquitecto y escritor italiano León Battista Alberti, quien en aras de reafirmar la condición humanista del arquitecto propuso los términos de "soliditas", "comoditas" y "voluptas".

8 MOSQUERA, Jemay. Epistemología y didáctica del proceso de enseñanza-aprendizaje en arquitectura y diseño industrial. En Revista científica Guillermo de Ockham. ISSN I794-I92X. julio - dic. de 2009, Universidad San Buenaventura. Cali. 2009, p 36.

M 65 REVISTA M VOL. 9 No.1. ENERO-JUNIO 2012 • FACULTAD DE ARQUITECTURA • UNIIERSIDAD SANTO TOMÁS COLOMBIA 
En ese sentido, la tendencia global predominante, evidenciada en una postura de los países industrializados que enfoca el desarrollo en función de la economía y las relaciones de poder (muy marcada en el caso colombiano por la influencia de los grupos de poder económico y político, las grandes desigualdades sociales y el deterioro de las identidades locales), pierde continuamente vigencia, en tanto no puede asegurar un progreso equitativo y justo hacia todos los miembros de las diferentes comunidades que habitan el territorio.

Como contratendencias que adquieren, cada vez, mayor fuerza los enfoques sociocultural y ambiental pueden contribuir a la fundamentación de principios universales y uniformidades empíricas que, frente a la evidente diversidad histórica y espacial, posibilitan la interpretación de constantes biológicas, psicológicas y societales, tales como, la interrelación y complementariedad de los diferentes componentes presentes en un contexto específico, el carácter transdisciplinar de los procesos de uso y ocupación del territorio y la formación integral por competencias.

Se busca entonces, combinar armónicamente lo global (universal) y lo local (regional), lo nuevo y lo viejo, lo lógico y lo dialógico, la innovación y la creatividad, las expectativas sociales y las condiciones del entorno, los procesos académicos, investigativos y de interacción social en una síntesis dialógica, fundamentada en una relación dialéctica de variables jerarquizadas. Las variables se agruparon en 3 categorías dentro de una síntesis integradora adaptativa representada en una articulación abierta, lógica y flexible del proceso de desarrollo de competencias y habilidades que aporta a las bases teóricas y prácticas necesarias para la formación investigativa. ${ }^{9}$ Estas categorías se encuentran relacionadas con: los procesos de enseñanza-aprendizaje, el desarrollo del pensamiento y las didácticas para la apropiación del conocimiento. Se determinaron como variables para los procesos de estudio: el conocimiento de sí mismo, el conocimiento de los demás y el conocimiento del entorno. Se determinaron como variables de enfoque: el enfoque sistémico, basado en la teoría general de los sistemas (TGS), los sistemas complejos adaptativos (SCA), el paradigma de la complejidad (C), y el pensamiento complejo (PC); el enfoque transdisciplinar, donde se reconocen las insuficiencias metodológicas, conceptuales e interpretativas de las disciplinas aisladas, y se aborda una visión integral de los fenómenos y conflictos que se han de investigar; y el enfoque didáctico, como sistema complejo. ${ }^{10}$ (Figura I)

\section{El Enfoque Sistémico y Holístico}

Ante la visión atomista y segmentada del mundo prevaleciente hasta finales del siglo XIX, se produjo la búsqueda de una nueva interpretación ecológica y espiritual de la realidad", la cual conllevó a la formulación de la teoría general de los sistemas ${ }^{12}$, representada en la reciprocidad e interrelación de los elementos que conforman cualquier sistema (proceso o hecho que se da en el universo) y en la necesidad del ser humano de adaptarse a los cambios que condicionan su devenir y evolución. Los sistemas fueron asumidos luego

9 MOSQUERA Jemay. Síntesis Integradora Adaptativa para el Proceso de Enseñanza - Aprendizaje de la Arquitectura. Memorias I Encuentro Latinoamericano: "Introducción a la enseñanza de la arquitectura: fundamentos y métodos". ISBN 978-958-8280-48-6, Universidad Nacional de Colombia Sede Manizales. 2010, p. 253

10 ZULUAGA, Lina, MOSQUERA, Jemay, HIGUERA Mercedes. Procesos de formación de líderes investigadores. Aplicados a los estudiantes del programa de arquitectura de la Universidad de Pamplona. Revista científica Guillermo de Ockham, ISSN 1794-192X. Universidad San Buenaventura. Cali. 2013, p. 43

II CADENAS Raíza, PACHANO Évelin, PEREIRA Eduardo Y TORRES Aura. "El paradigma de la complejidad. Un cadáver exquisito". Revista Electrónica de Epistemología de Ciencias Sociales. Universidad de Chile: Facultad de Ciencias Sociales, No. 14. ISSN 07I7-554X, 2002

12 VON BERTALANFFY Ludwig. Teoría General de los Sistemas. Décima edición, Buenos Aires, Editorial Fondo de Cultura económica, 1995. Pp. 2-22. 
como sistemas complejos adaptativos, caracterizados por temer propósito, capacidad de aprendizaje, ajuste, autoorganización ${ }^{13}$ y adaptabilidad a las condiciones del entorno ${ }^{14}$.

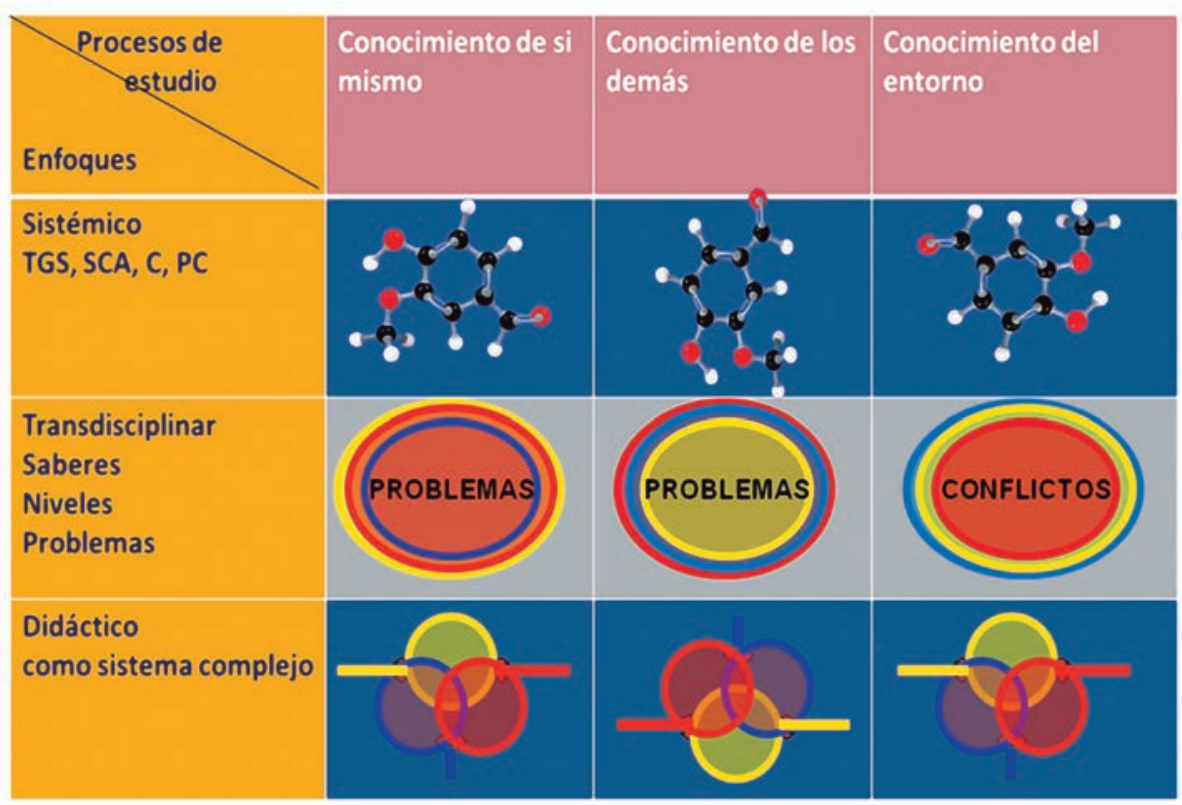

Esta visión integradora y holística conduce a la formulación del Paradigma de la Complejidad $^{15}$, en el que a partir de los principios de incompletud e incertidumbre se pretende lograr la articulación de conceptos antagónicos y la organización del conocimiento en una relación homogeneidad-heterogeneidad ${ }^{16}$, como prerrequisitos para llegar al Pensamiento Complejo. Así el pensamiento complejo articula la lógica y la dialógica, la argumentación y las estrategias cognitivas, para ayudarnos a aprender y captar la complejidad, para dar sentido y conferir significado tanto a lo global como lo local expresado “(...) por la doble pareja pensar global/actuar local, pensar local/actuar global”|17. De esta manera, los procesos de la arquitectura son vistos como recursivamente auto-constructivos, con una multiplicidad de factores, indicios, símbolos y señales en permanente interacción e interdependencia, que condicionan y son condicionados y que podemos visualizar desde una diversa gama de puntos de vista (producto de la subjetividad de cada ser humano) y de nuevas relaciones emergentes entre esos fenómenos en contextos espacio temporales específicos. ${ }^{18}$

La propuesta para consolidar el enfoque sistémico en el proceso de enseñanza - aprendizaje de la arquitectura, está representada en la interpretación de los objetos y las configuraciones espaciales de la arquitectura como sistemas complejos adaptativos, dinámicos, evolutivos y, por qué no efímeros, compuestos por una enorme cantidad de componentes interactuantes simples, capaces de intercambiar información con su entorno y de adaptar su estructura

3 MATURANA, Humberto. El sentido de lo humano, Primera edición, Santiago de Chile, Dolmen Ediciones S. A., 1997. Pp. 5-I5.

14 HOLLAND, John Henry. Complex Adaptive Systems. Daedalus, Vol. I2I, 1992. Pp. 17-30.

I5 MORIN, Édgar. Introducción al pensamiento Complejo, Barcelona, Gedisa, 1997. Pp. 3-I2.

I6 MORIN, Édgar. El Método V. La humanidad de la humanidad, La identidad humana. ISBN. 84-376-2047-3. Editorial Cátedra S.A. Madrid, 2003.

I7 MORIN, Édgar. CIURANA, Emilio. y MOTTA Raúl. Educar en la era planetaria. El pensamiento complejo como un método de aprendizaje en el error y la incertidumbre humana. I a . Ed. España, U de Valladolid, Editorial UNESCO, 2003, p. 38.

18 MOSQUERA, Jemay. "Arquitectura y Complejidad". Revista Ambiental Agua, Aire y Suelo. Facultad de Ingenierías y Arquitectura. ISSN 1900-9I78 Julio - diciembre de 2007, Vol. 2, N I, año 2. Universidad de Pamplona. 2007, pp. 6-8.
Figura I. Variables de procesos de estudio y de enfoque. Fuente: Mosquera, 2010 
Tabla I. Matriz Sistémica para talleres de diseño y proyectos de investigación. Fuente: Grupo de Investigación Gestión Integral del Territorio - GIT interna a partir de tales interacciones, para la configuración de proyectos arquitectónicos compuestos con niveles superiores de complejidad. Lo anterior se ve expresado en la matriz sistémica para talleres de diseño y proyectos de investigación del programa de arquitectura de la Universidad de Pamplona en la que se parte del enfoque sistémico del Ministerio de Ambiente, Vivienda y Desarrollo Territorial articulado a las temáticas definidas por la Sociedad Colombiana de Arquitectos para las Bienales de Arquitectura y complementada nociones propias del programa de Arquitectura (Tabla I).

\begin{tabular}{|c|c|c|c|c|c|}
\hline \multicolumn{6}{|c|}{ MATRIZ SISTÉMICA PARA TALLERES DE DISEÑO Y PROYECTOS DE INVESTIGACIÓN EN ARQUITECTURA } \\
\hline \multirow{4}{*}{ NÚCLEOS SISTÉMICOS } & \multicolumn{5}{|c|}{ SISTEMAS DEL TERRITORIO } \\
\hline & AMBIENTAL & CULTURAL & SOCIAL & ECONÓMICO & POLÍTICO \\
\hline & \multicolumn{5}{|c|}{ Principios de los núcleos sistémicos del territorio } \\
\hline & \begin{tabular}{|l|} 
Hábitat \\
sostenible
\end{tabular} & $\begin{array}{c}\text { Valoración, } \\
\text { recuperación y } \\
\text { fortalecimiento de } \\
\text { Identidades culturales }\end{array}$ & $\begin{array}{l}\text { Equidad e } \\
\text { inclusión }\end{array}$ & $\begin{array}{l}\text { Competitividad a } \\
\text { escala humana }\end{array}$ & $\begin{array}{c}\text { Gobernabilidad y } \\
\text { gobernanza }\end{array}$ \\
\hline \multirow[t]{2}{*}{ NÚCLEOS PROBLÉMICOS } & \multicolumn{5}{|c|}{ NÚCLEOS PROBLÉMICOS } \\
\hline & \begin{tabular}{|l|} 
Insostenibilidad, \\
amenaza, \\
vulnerabilidad y \\
riesgo, \\
contaminación y \\
deterioro, \\
naturaleza \\
como objeto de \\
derechos
\end{tabular} & $\begin{array}{l}\text { pérdida de } \\
\text { identidades y bajo } \\
\text { sentido de } \\
\text { pertenencia, } \\
\text { transculturización, } \\
\text { desterritorialización, } \\
\text { pocos espacios para } \\
\text { manifestaciones } \\
\text { culturales, deterioro y } \\
\text { poca valoración del } \\
\text { patrimonio material e } \\
\text { inmaterial }\end{array}$ & $\begin{array}{l}\text { Exclusión, } \\
\text { pobreza, } \\
\text { desigualdad, } \\
\text { segmentación, } \\
\text { necesidades } \\
\text { básicas } \\
\text { insatisfechas, bajo } \\
\text { índice de } \\
\text { desarrollo } \\
\text { humano, poca } \\
\text { felicidad }\end{array}$ & $\begin{array}{l}\text { Marginalidad, } \\
\text { informalidad, } \\
\text { distribución } \\
\text { inequitativa de } \\
\text { recursos, baja o } \\
\text { nula inserción en } \\
\text { los mercados, } \\
\text { poca atracción e } \\
\text { incorporación de } \\
\text { tecnología, baja } \\
\text { competitividad. }\end{array}$ & $\begin{array}{l}\text { Falta de } \\
\text { transparencia y } \\
\text { credibilidad, baja } \\
\text { participación de } \\
\text { actores sociales, } \\
\text { baja gobernabilidad } \\
\text { y gobernanza, } \\
\text { inexistencia de } \\
\text { normativa o baja } \\
\text { aplicación de } \\
\text { normativa existente }\end{array}$ \\
\hline \multicolumn{6}{|l|}{ Teoría, historia y crítica } \\
\hline \multicolumn{6}{|l|}{ Diseño urbano y paisajístico } \\
\hline \multicolumn{6}{|l|}{ Hábitat popular } \\
\hline \multicolumn{6}{|l|}{ Proyecto arquitectónico } \\
\hline \multicolumn{6}{|l|}{ Recuperación del patrimonio } \\
\hline \multicolumn{6}{|l|}{ Tecnológico constructivo } \\
\hline Ordenamiento territorial & & & & & \\
\hline
\end{tabular}

\section{LA NOCIÓN TRANSDISCIPLINAR}

En cuanto al proceso disciplinar, se propone evidenciar las categorías de la arquitectura como reflejo del pensamiento y las características multidimensionales de la época en que se desarrolla. Dicho proceso trasciende las particularidades individuales de la arquitectura y está representado en sus vínculos humanísticos con las ciencias sociales (sociología, antropología, psicología, economía y política, entre otras), vínculos tecnológicos con las ciencias exactas (física, matemáticas y geometría, entre otras), las ciencias naturales (química y biología) y el saber propio de la técnica; así como vínculos con la estética, la lógica y la dialógica.

Estos vínculos están, a su vez, enmarcados en las metodologías generales de los procesos de diseño, las cuales responden a tres pasos clave: el conocimiento de sí mismo, representado en el acercamiento a las formas del ser, tener, hacer y estar con el apoyo (si se desea) de expertos (líderes comunitarios con experiencia y capacidades pedagógicas); el conocimiento de los demás, representado en la comprensión de las necesidades existenciales del otro, de la complementariedad y la unidad en la diversidad; $y$ el conocimiento del entorno, representado en la valoración y apropiación de las potencialidades que ofrece el entorno natural y antrópico para el bienestar humano. 
La transdisciplinariedad nace de la noción del conocimiento como un sistema abierto y evolutivo, reconoce las insuficiencias metodológicas, conceptuales e interpretativos de las disciplinas aisladas, aborda una visión integral de los fenómenos y conflictos que se han de investigar, tiene en cuenta los seres involucrados ${ }^{19} y$ articula ciencias y conocimientos para dar respuesta a los conflictos de uso del territorio desde la unidad de la comprensión ${ }^{20}$, en un diálogo recíproco de conocimientos y saberes. ${ }^{21}$

Dado el reconocimiento de los diversos saberes que giran en torno a las ciencias sociales y humanas, naturales, exactas y los saberes propios de las técnicas, la ética, la lógica y la estética como apoyo de los saberes de la arquitectura, se hace necesario reflexionar sobre sus objetos de estudio y sus metodologías de aproximación al conocimiento, con el fin de poder sustentar, a su vez, las áreas de conocimiento propias del arquitecto. Para ello, se deja de considerar a la arquitectura como una práctica aislada y autónoma, se hace énfasis en sus correlaciones socio-naturales con los seres humanos y el entorno para lograr un equilibrio basado en la convergencia e interacción de diversas corrientes de pensamiento, como conditio sine qua non, donde se produce adecuadamente la creación arquitectónica.

En un intento por articular los vínculos disciplinares y los pasos de las metodologías de diseño, en el programa de arquitectura de la Universidad de Pamplona se parte de un enfoque multidisciplinario (donde la categoría y la especialidad son propias) y se pasa a un enfoque Interdisciplinario (donde las disciplinas asumen factores de unidad pero conservan los límites propios), para luego llegar a un enfoque transdisciplinar, representado en la conjunción transversal de conocimientos que rebasa los límites disciplinares y pone, por encima de todo, la interpretación de una realidad compleja y la solución de problemas y conflictos inmersos en dicha realidad universal-local. Lo anterior se ve reflejado en la integración que se da desde primer semestre de contenidos programáticos propios del programa de arquitectura con materias de otros programas, tales como diseño industrial e ingeniería civil y con materias transversales institucionales. Además, se han puesto en marcha proyectos de investigación tendientes a la solución de problemas reales, en los que confluyen estudiantes del programa de arquitectura, con estudiantes de diferentes programas de la institución.

\section{LA BIOPEDAGOGÍA Y LA DIDÁCTICA}

En cuanto a los procesos académicos y su relación con la pedagogía y la didáctica, si bien la educación se da a través de procesos conscientes o inconscientes, la pedagogía y la didáctica son siempre conscientes e intencionales: "la pedagogía aborda la pregunta de cómo educar/ aprender desde un enfoque antropológico y filosófico, la didáctica aborda la pregunta de cómo enseñar/aprender desde un enfoque histórico y práctico". ${ }^{22}$

Ante la necesidad de aprender a aprender, aprender a ser, aprender a desaprender, aprender a vivir, aprender a convivir, aprender a servir y aprender a disfrutar a lo largo de toda la vida, es evidente que más allá de la formación de profesionales en arquitectura, los es-

19 HURNI, Hans y WIESMANN, Urs. Investigación transdisciplinar en el contexto del desarrollo: iFórmula vacía o necesidad? CDMA-IGUB. Suiza. 2003. p. 6

20 NICOLESCU, Basarab. La transdisciplinariedad. Una nueva visión del mundo. Traducido del francés por Consuelo Falla. México. S.p. 1999.

21 MORIN, Édgar. Carta de la transdisciplinariedad de Édgar Morin. Portugal. S.p. 1994

22 MOSQ UERA, Jemay. Epistemología y didáctica del proceso de enseñanza-aprendizaje en arquitectura y diseño industrial. En Revista científica Guillermo de Ockham. ISSN 1794-192X. jul. - dic. de 2009, Universidad San Buenaventura. Cali. 2009, p. 42 
Figura 2. Articulación de los procesos misionales institucionales. Fuente: Grupo de Investigación Gestión Integral del Territorio - GIT fuerzos deben ser dirigidos a la articulación de la docencia, la investigación y la interacción social (Figura 2), para responder pertinentemente a las nuevas demandas ${ }^{23}$ y necesidades cambiantes $^{24}$ en la sociedad del conocimiento. ${ }^{25}$

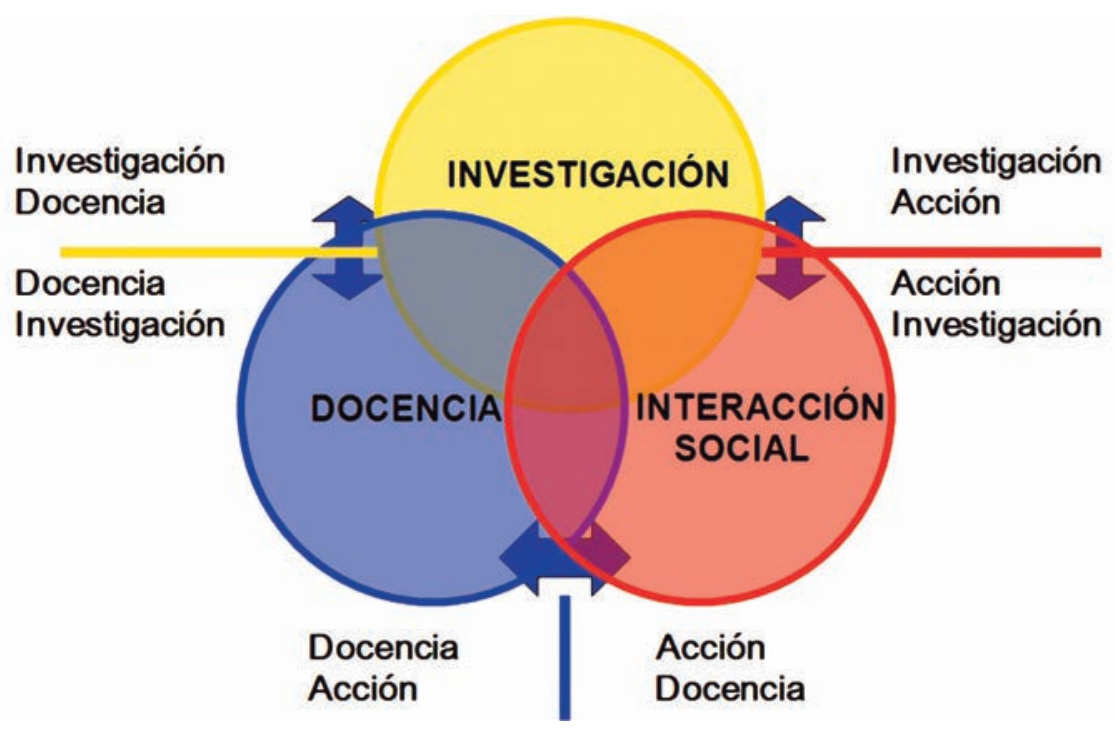

En ese orden de ideas, la bio-pedagogía asume al aprendizaje como actividad flexible y adaptativa de los organismos vivos y ha permitido reconocer la necesidad individual y social de persistencia y constancia en los procesos de aprendizaje. De esta manera, la dinámica de aprender a lo largo de la vida se convierte en una propiedad emergente y necesidad vital que permite direccionar las actividades cognitivas para dar respuestas pertinentes a las demandas sociales y laborales, "como proceso de autoorganización, desde el plano biofísico hasta el de los ámbitos sociales". ${ }^{26}$

La bio-pedagogía se enfoca hacia la promoción del aprendizaje, la generación de redes de investigación y comunidades de aprendizaje en torno a proyectos de académicos de extensión-investigación que respondan a las necesidades de las diversas realidades y a las propias necesidades biológicas de los estudiantes, al asumir que la pedagogía debe ser consciente de que si bien no es la única instancia educativa, no puede renunciar a “(...) la función de crear conscientemente experiencias de aprendizaje, reconocibles como tales por los sujetos implicados"27. Tales proyectos, promueven la formación de competencias investigativas, las cuales ofrecen insumos para desarrollar funciones meta-cognitivas con las que los estudiantes aprenden sobre su propio proceso de aprendizaje, analizan críticamente y problematizan la realidad para formular propuestas tendientes a la resolución de conflictos de uso del territorio en niveles de ejecución posibles. La creación de redes de investigación y comunidades de aprendizaje con docentes, estudiantes y egresados, fortalece el desarrollo de espacios pedagógicos en los cuales se crean nuevas condiciones para la autoorganización de las experiencias de aprendizaje, en las que "educar es crear, realizar, y validar en la convivencia, un modo particular de convivir”. ${ }^{28}$

23 DIDRIKSSON, Axel. Contexto global y regional de la educación superior en América Latina y el Caribe, 2008.

24 HORRUITTINER, Pedro. El reto de la transformación curricular. Revista lberoamericana de Educación. 40(3), 2006. Pp. $1-13$.

25 DE MIGUEL, Jesús Manuel. Universidad y democracia. (3a Ed). Cádiz, España: Fundación Municipal de Cultura, 2003

26 ASSMANN, Hugo. Placer y Ternura en la Educación, Hacia una sociedad aprendiente. España: Nancea, S. A. 2002 , p. 27.

27 Ibídem p. 26

28 MATURANA, Humberto. Transformación en la convivencia. Comunicaciones Noreste Ltda. ISBN 956-7802-29-7. Santiago de Chile, 2004, p. 147 
El reconocimiento de la complejidad y de la diversidad del proceso de formación ${ }^{29}$, referido a la incorporación de la multiplicidad de enfoques, actividades y herramientas metodológicas, como elemento estructural para la interpretación de las realidades permite concebir y desarrollar planes de estudios integrados que reflejen

“(...) los intereses, necesidades y capacidades de los estudiantes, se extienden más allá del salón de clases, otorgan sentido de responsabilidad social, comparten una visión, estimulan la cooperación para disfrutar de trabajar juntos y estimulan una cultura de sabiduría, una conciencia indagatoria". ${ }^{30}$

Las actitudes y aptitudes se deben articular a un aprendizaje atractivo y placentero en el que "La cuestión sobre calidad cognitiva y social de la educación se debe afrontar, primordialmente, desde un eje pedagógico, es decir, a partir de la experiencia del placer de estar conociendo"31 en el que las experiencias formadoras se convierten en significativas.

Las experiencias investigativas a partir de matrices sistémicas de delimitación y articulación de elementos metodológicos y procedimentales aseguran la configuración de espacios de aprendizaje y "ecologías cognitivas de ambientes que propician experiencias de conocimiento" 32 , formación permanente y pertinente al desarrollo de la arquitectura y de la sociedad.

Por su parte, la didáctica se fundamenta en los actos educativos y hace uso de las transformaciones espacio-temporales del saber, representadas primordialmente en las trasposiciones didácticas de reconceptualización y recontextualización de los saberes, ${ }^{33}$ para sentar sus principios de formación integral, aprendizaje basado en problemas y formación en investigación ${ }^{34}$. El primero, está relacionado con la diversidad del sujeto y la formación intelectual, socio-humanística y profesional; el segundo, con la búsqueda de solución a problemas y conflictos reales $y$, el tercero, con los métodos y la estructura metodológica necesaria para sistematizar o desarrollar nuevos conocimientos

Del mismo modo, se precisa tener en cuenta los principales paradigmas que influyen en el proceso de aprendizaje para abordar y aplicar modelos pedagógicos emergentes que articulen armónicamente el conocimiento y la práctica, modelos centrados en procesos y basados en el aprendizaje significativo ${ }^{35}$, en la transacción participativa y en la subjetividad crítica.

En Colombia, sobre la base de normas generales, cada programa hace su propio diseño curricular con criterios de calidad, cobertura, pertinencia y eficiencia, así como de flexibilidad e integralidad. En la Universidad de Pamplona, el programa de arquitectura asume estos criterios desde la apropiación consciente del aprendizaje en un intento de complementar adecuadamente la conducta instintiva propia de los seres biológicos.

29 MORIN, Édgar. El Método V. La humanidad de la humanidad, La identidad humana. ISBN. 84-376-2047-3. Editorial Cátedra S.A. Madrid. 2003.

30 GALLEGOS, Ramón. Educación Holística: Pedagogía del amor universal. México. PAX México, 1999, p. 177

3I Ibídem 2I, p. 28

32 Ibídem 21 , p. 23

33 CHEVALLARD, Yves. La transposición didáctica. Buenos Aires: Aique. ISBN: 950-70I-380-6, I99I.

34 lbídem 17, p. 42

35 AUSUBEL, David Paul, NOVAK, Joseph, HANESIAN, Helen. Psicología Educativa: Un punto de vista cognoscitivo. $2^{\circ}$ Ed. Trillas, ISBN: 97896824I3346. México, 1983. 


\section{EL CONOCIMIENTO COMO EJE ARTICULADOR DE LOS PROCESOS DE DISEÑO}

Los procesos de diseño se desarrollan de múltiples maneras que involucran diferentes aproximaciones conceptuales y procedimentales. No obstante, todas las formas aplicadas de diseño se caracterizan por tener tres componentes similares que sirven de base para su desarrollo efectivo, el conocimiento de sí mismo, el conocimiento de los demás y el conocimiento del entorno.

El conocimiento de sí mismo, permite el acercamiento a las formas del ser, tener, hacer y estar con el apoyo (opcional pero recomendado) de expertos, lo cuales no solamente pueden ser arquitectos experimentados, sino también líderes comunitarios con experiencia y capacidades de organización social. Este tipo de conocimiento permite dilucidar que las decisiones que se toman diariamente son generalmente producto de las emociones pasajeras (en mayor grado) y del razonamiento (en menor grado).

Si a estos dos elementos, se les agrega los sentimientos duraderos, los cuales son resultado de uno o más estímulos a través de los cuales el consciente tiene acceso al subconsciente, es posible modelar la conducta (expresada en actitudes y cualidades) y, por consiguiente, las actitudes, entendidas como la expresión individualizada de los principios o comportamientos acordados en consenso. De esta manera, se pueden cultivar los valores, es decir, los principios que facilitan conformación de actitudes correctas y asertivas, y se pueden lograr decisiones conscientes para los procesos de diseño.

La conducta por instinto está representada en un esquema de respuesta predeterminado desde los instintos de conservación personal, conservación de la especie, el trabajo en equipo y la competitividad. La conducta por consciencia requiere de la integración de la información y la conformación de esquemas de respuesta en determinado tiempo y velocidad. Estos procesos se dan en dos niveles, el consciente y el subconsciente y están condicionados por la necesidad de interpretar conceptos y aplicar conocimientos.

Los ideales de la consciencia del ser humano se relacionan con el ser (desde la visión disciplinar y transdisciplinar), el saber (mediante los conocimientos aplicados), el hacer (por medio de procesos y etapas de desarrollo procedimental), el tener (con productos concretos evaluables) y el servir (a partir de la utilidad social), así como con los rasgos de la personalidad, tales como, actitud y aptitud, para la generación de condiciones de éxito en el desarrollo y aplicación de competencias y capacidades profesionales.

Se han definido entonces cuatro niveles de consciencia relacionados con la formación por competencias que promueve el Ministerio de Educación Nacional de Colombia:

Inconscientemente incompetente o estado a partir del cual el ser humano refleja la primacía de condiciones instintivas conjuntamente con la ausencia de aptitudes y actitudes para el desarrollo de actividades académicas disciplinares y profesionales.

Conscientemente incompetente o estado a partir del cual el ser humano refleja la ausencia voluntaria de aptitudes y actitudes para el desarrollo de actividades académicas disciplinares y profesionales. 
Inconscientemente competente o estado a partir del cual el ser humano refleja la baja apropiación de la necesidad de interpretar conceptos y aplicar procesos para la generación de conocimientos, pero al mismo tiempo cuenta con aptitudes y actitudes para un desarrollo relativo y/o parcializado de actividades académicas disciplinares y profesionales.

Conscientemente competente o estado a partir del cual el ser humano refleja una rigurosa necesidad de interpretar conceptos y aplicar procesos para la generación de conocimientos $y$, al mismo tiempo, está relacionado con la presencia de aptitudes y actitudes para un desarrollo coherente y articulado de actividades académicas disciplinares y profesionales (Figura 3).

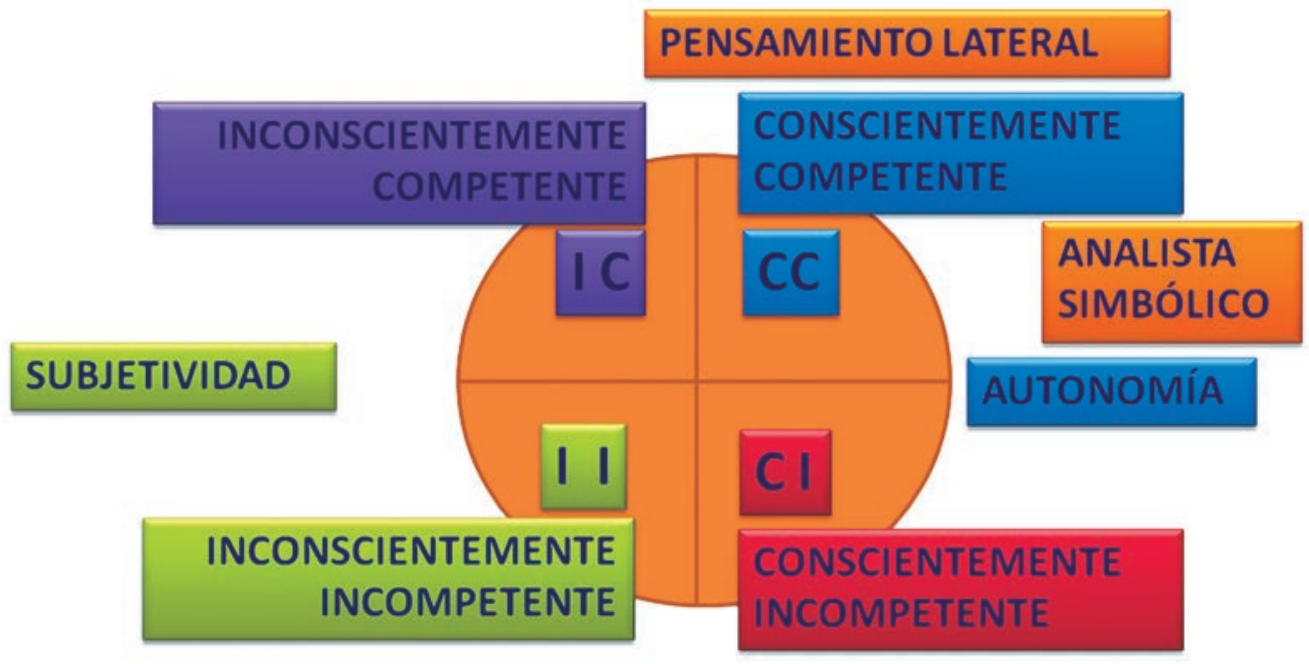

El conocimiento de los demás, involucra la interpretación de las necesidades existenciales del otro, de las relaciones de especialización y complementariedad, de la unidad en la diversidad y de la mutualidad, asumida como la interrelación entre la sensibilidad y la construcción de alteridad. Asimismo, está asociado a la predisposición al cambio y a la predisposición (positiva y negativa) que posee el ser humano en el desarrollo de sus relaciones interpersonales y que le permiten avanzar en la identificación de problemas y la gestión de conflictos sentidos de la comunidad.

Por último, el conocimiento del entorno está relacionado con la valoración y apropiación de las potencialidades que ofrece el contexto natural y antrópico para el bienestar humano. En ese sentido, la concepción de territorio es abordada desde las relaciones que se dan en él y que configuran una estructura territorial sistémica de múltiples interacciones: relaciones del ser humano con la naturaleza que conforman el sistema ambiental, relaciones de producción e intercambio que conforman el sistema económico, relaciones de poder que conforman el sistema político administrativo y relaciones del ser humano con su comunidad y su historia que conforman los sistemas social (generalizante) y cultural (particularizante). El estudio de dichas relaciones debe responder a un enfoque integral de aproximación sinérgica dirigido a proteger y respetar el espacio natural “(...) a partir del uso adecuado y de la apropiación consciente". ${ }^{36}$

36 MOSQUERA, Jemay. "Planificación Ambiental y Arquitectura Sostenible". En Revista Ambiental Agua, Aire y Suelo. ISSN 1900-9|78. Vol. I, No, 2, Universidad de Pamplona. 2006, p. 92
Figura 3. Niveles de competencia Consciente Fuente: Grupo de Investigación Gestión Integral del Territorio - GIT 


\section{CONCLUSIONES}

La formación en arquitectura trasciende la formación disciplinar, involucra la responsabilidad de preparar estudiantes capaces y conscientes de la necesidad de autorregular sus procesos de aprendizaje con competencias básicas para la investigación que les permitan evolucionar en su desempeño profesional.

El proceso de enseñanza - aprendizaje de la arquitectura requiere de nuevos esquemas mentales de conceptualización, análisis y valoración crítica y comparada de los hechos arquitectónicos que permitan estudiar e interpretar la heterogeneidad, interdependencia y complementariedad de los procesos y conflictos presentes en el espacio habitado y propendan por la formulación de propuestas encaminadas a asegurar un desarrollo integral del territorio.

Se propone abordar el proceso de formación en arquitectura por medio de una síntesis integradora adaptativa representada en una articulación abierta y flexible del proceso de desarrollo de competencias y habilidades, cuya estructura hermenéutica se fundamenta en la subjetividad del ser humano y posibilita la definición de diversos métodos y aproximaciones metodológicas en la enseñanza de la arquitectura, las cuales se adaptan fácilmente a los diversos niveles de complejidad de las actividades y procesos académicos (ejercicio puntual, proyecto, semestre, periodo formativo y estructura académica) desde los diferentes sistemas (cultural, social, ambiental, económico y político) del desarrollo, así como desde los procesos de diseño y los procesos pedagógicos y didácticos.

Las actividades puntuales que se derivan de los aportes enunciados son las siguientes:

- El desarrollo de currículos integrados al interior del programa de arquitectura y el inicio de complementariedades investigativas con carreras afines a la arquitectura para la solución de problemas y gestión de conflictos reales.

- La implementación de mecanismos para la articulación y realización de trabajos estudiantiles conjuntos entre programas de pregrado y entre programas de pregrado y posgrado a partir de las convocatorias internas de investigación.

- La promoción de la integración activa y real en el aula de los procesos de docencia, investigación e interacción social a partir de las convocatorias internas de investigación.

- El respeto por el conocimiento, en una permanente actitud de contraste de conceptos, formulación de problemas reales y búsqueda de soluciones innovadoras y útiles a la sociedad por medio de ejercicios proyectuales articulados a diferentes materias del plan de estudios.

- La configuración de espacios pedagógicos, redes de investigación y comunidades de aprendizaje, concebidos como escenarios para los procesos académicos, de investigación e interacción social en un marco equitativo de respeto y reconocimiento de todos los actores participantes. Entre ellos se destacan la articulación de diferentes materias del plan de estudios al taller de diseño, el taller vertical ínter-semestral que ha generado las bases para una propuesta programática, operativa y tectónica propia de los proyectos arquitectónicos del programa de arquitectura, los semilleros de investigación que se generan en torno a proyectos concretos, los trabajos de se- 
mestre y de grado articulados a trabajos de posgrados y a proyectos investigativos, los vigías del patrimonio en los que participan estudiantes y egresados del programa de arquitectura.

De esta manera, se busca el paso de una condición inconscientemente competente a procesos formativos conscientemente competentes que propenden por cualificar la enseñanza y el aprendizaje de la arquitectura, por fomentar en los futuros arquitectos el uso adecuado y la apropiación consciente del territorio en función de su proyección al medio y de la satisfacción de las necesidades reales de la comunidad.

\section{BIBLIOGRAFÍA}

ASSMANN, Hugo. Placer y Ternura en la Educación, Hacia una sociedad aprendiente. España: Nancea, S. A. 2002, pp.23-28.

AUSUBEL, David Paul, NOVAK, Joseph, HANESIAN, Helen. Psicología Educativa: Un punto de vista cognoscitivo. $2^{\circ}$ Ed. Trillas, ISBN: 97896824 I3346. México, I983. 623 p.

BAJTIN, Mijail. Las formas de tiempo y del cronotopo en la novela, ensayos de poética histórica. Teoría y estética de la novela; Madrid, Taurus, 1989, pp. 237-409.

CADENAS Raíza, PACHANO Evelin, PEREIRA Eduardo Y TORRES Aura. "El paradigma de la complejidad. Un cadáver exquisito”. Revista Electrónica de Epistemología de Ciencias Sociales. Universidad de Chile: Facultad de Ciencias Sociales, No.. I4. ISSN 07I 7-554X, 2002.

CHEVALLARD, Yves. 1991. La transposición didáctica. Buenos Aires: Aique. ISBN: 95070I-380-6, 199I. $196 \mathrm{p}$.

DE MIGUEL, Jesús Manuel. Universidad y democracia. ( $3^{\mathrm{a} e d}$ ). Cádiz, España: Fundación Municipal de Cultura. 2003

DIDRIKSSON, Axel. 2008. Contexto global y regional de la educación superior en América Latina y el Caribe. Disponible en línea en www.iesalc.unesco.org.ve

GALLEGOS, Ramón. Educación Holística: Pedagogía del amor universal. México. PAX México, 1999, p. 177.

GUBA, Egon. LINCOLN, Yvonna. "Competing paradigms in qualitative research" en Denzin, N. Lincoln (comps.): Handbook research, Sage Publications, California, 1994. pp. I05-I I 7.

HOLLAND, John Henry. Complex Adaptive Systems. Daedalus, vol. I2I, 1992. Pp. 17-30, Winter.

HORRUITTINER, Pedro. El reto de la transformación curricular. Revista lberoamericana de Educación. 40(3), 2006. p. I-I3. Recuperado del 25 de mayo del 2009, de http://www. rieoei.org/deloslectores/I524Silva.pdf

M 75 REVISTA M VOL. 9 No.1. ENERO-JUNIO 2012 • FACULTAD DE ARQUITECTURA • UNIVERSIDAD SANTO TOMÁS COLOMBIA 
HURNI, Hans y WIESMANN, Urs. Investigación transdisciplinar en el contexto del desarrollo: iFórmula vacía o necesidad? CDMA-IGUB. Suiza. 2003. 6 p. (mimeo)

JONES, John Christopher. Diseñar el diseño. Barcelona, Gustavo Gili, I edición. 1984.

MATURANA, Humberto. El sentido de lo humano, Primera edición, Santiago de Chile, Dolmen Ediciones S.A., 1997. pp. 5-I5.

MATURANA, Humberto. Transformación en la convivencia. Comunicaciones Noreste Ltda. ISBN 956-7802-29-7. Santiago de Chile, 2004. 285 p.

MORIN, Édgar. Carta de la transdisciplinariedad de Édgar Morin. Portugal. S.p. 1994

MORIN, Édgar. Introducción al pensamiento Complejo, Barcelona, Gedisa, 1997. pp. 3-I2.

MORIN, Édgar. El Método V. La humanidad de la humanidad, La identidad humana. ISBN. 84-376-2047-3. Editorial Cátedra S.A. (Grupo Amaya) Madrid. 2003. 342 p.

MORÍN, Edgar. CIURANA, Emilio y MOTTA Raúl. Educar en la era planetaria. El pensamiento complejo como un método de aprendizaje en el error y la incertidumbre humana. $I^{\text {a }}$. Ed. España, U de Valladolid, Editorial UNESCO, 2003. pp. 38-80.

MOSQUERA, Jemay. "Planificación Ambiental y Arquitectura Sostenible". En Revista Ambiental Agua, Aire y Suelo. ISSN 1900-9178. Vol. I, No. 2, Universidad de Pamplona. Pamplona, 2006, pp. 87-93

MOSQUERA, Jemay. Arquitectura y Complejidad. En Revista Ambiental Agua, Aire y Suelo. ISSN 1900-9I78 Julio - dic. de 2007, Vol. 2, No. I, año 2. Universidad de Pamplona. Pamplona, 2007. pp. 3-10.

MOSQUERA, Jemay. Epistemología y didáctica del proceso de enseñanza-aprendizaje en arquitectura y diseño industrial. En Revista científica Guillermo de Ockham. ISSN I794192X. julio - dic de 2009, Universidad San Buenaventura. Cali. 2009, pp. 33-49. 
MOSQUERA Jemay. Síntesis Integradora Adaptativa para el Proceso de Enseñanza Aprendizaje de la Arquitectura. Memorias I Encuentro Latinoamericano: "Introducción a la enseñanza de la arquitectura: fundamentos y métodos". ISBN 978-958-8280-48-6, Universidad nacional de Colombia Sede Manizales. 2010, pp. 237-259

MOSQUERA, Jemay. Síntesis dialógica para la formación en arquitectura. Memorias 5 Seminario Internacional Arquitectonics Network en América, the Project as research, research as Project, ISSN 978-958-8477-32-9. Universidad Santo Tomás, Bucaramanga. 20I2, pp. 24-27

MUNTAÑOLA, Josep. Arquitectura, educación y dialogía social. En Revista española de de pedagogía, año LXII. No. 228, Universidad Politécnica de Catalunya, España. 2004, p $221-228$

NICOLESCU, Basarab. La transdisciplinariedad. Una nueva visión del mundo. Traducido del francés por Consuelo Falla. México. S.p. 1999.

SALDARRIAGA, Alberto. Aprender Arquitectura. Un manual de supervivencia. En: Colombia 1966. Ed. Fundación Corona ISBN: 958-9054-4 v. I70 p. 1996. pp 22-23

VON BERTALANFFY Ludwig. Teoría General de los Sistemas. Décima edición, Buenos Aires, Editorial Fondo de Cultura económica, 1995. pp. 2-22.

ZULUAGA, Lina, MOSQUERA, Jemay, HIGUERA Mercedes. Procesos de formación de líderes investigadores. Aplicados a los estudiantes del programa de arquitectura de la Universidad de Pamplona. Revista científica Guillermo de Ockham, ISSN 1794-192X. Universidad San Buenaventura. Cali. 2013, pp. 37-47 